\title{
Association of adjuvant aromatase inhibitor with cataract risk in postmenopausal women with breast cancer
}

\author{
Han Chen ${ }^{1}$, Zhi-Ming Shao ${ }^{2}$, Ke-Da Yu' ${ }^{2}$ Ge-Zhi Xu' ${ }^{1}$ \\ ${ }^{1}$ Department of Ophthalmology, Eye, Ear, Nose and Throat Hospital, Fudan University, Shanghai 200031, China; ${ }^{2}$ Department of Breast Surgery, \\ Shanghai Cancer Center, Fudan University, Shanghai 200032, China \\ Contributions: (I) Conception and design: KD Yu, H Chen; (II) Administrative support: ZM Shao, GZ Xu; (III) Provision of study materials or \\ patients: KD Yu, ZM Shao; (IV) Collection and assembly of data: KD Yu, ZM Shao; (V) Data analysis and interpretation: KD Yu, H Chen, GZ Xu; (VI) \\ Manuscript writing: All authors; (VII) Final approval of manuscript: All authors. \\ Correspondence to: Ke-Da Yu, MD, PhD. Department of Breast Surgery, Fudan University Shanghai Cancer Center, Shanghai Medical College, Fudan \\ University, 270 Dong An Road, Shanghai 200032, China. Email: yukeda@163.com; Ge-Zhi Xu, MD, PhD. Department of Ophthalmology, Eye, Ear, \\ Nose and Throat Hospital, Fudan University, 83 Fenyang Road, Shanghai 200031, China. Email: xugezhi@gmail.com.
}

\begin{abstract}
Background: Aromatase inhibitor (AI) is a cornerstone drug for endocrine therapy of postmenopausal hormone receptor-positive breast cancer. The relationship between AI medication and subsequent cataract risk is yet inconclusive.
\end{abstract}

Methods: A total of 1,697 postmenopausal, early-staged, and hormone receptor-positive breast cancer patients from the prospectively-maintained database of the Breast Surgery Department at Fudan University Shanghai Cancer Center were included, with 542 patients received no endocrine therapy and 1,155 treated with AI only. We explored the influence the use of AI or not and the duration of AI on the subsequent incidence of cataract.

Results: A total of 146 (8.6\%) cataracts were observed in the whole study population. The incidence of cataract is highly related to age, with incidence of cataract in patients $<50$ years old, 50-60 years, $\geq 60$ years was $2.9 \%, 6.9 \%$, and $13.3 \%$, respectively $(\mathrm{P}<0.001)$. There was no significant relationship between adjuvant AI use and cataract (7.4\% in no endocrine group vs. 9.2\% in the AI group) with an adjusted hazard ratio of 1.20 (95\% confidence interval: $0.8-1.7, \mathrm{P}=0.30$ ). The incidence of cataract in patients with long-term AI (more than 5 years) could be high up to $14.7 \%$, but without statistical significance compared to those the shorter duration $(\mathrm{P}=0.52)$.

Conclusions: There is no significant association between use of AI and cataract in postmenopausal breast cancer patients. Of note, age is an important risk factor for cataract and it is necessary to surveil the eye health in postmenopausal elderly women.

Keywords: Breast cancer; aromatase inhibitor (AI); endocrine therapy; cataract

Submitted Jan 08, 2020. Accepted for publication Feb 10, 2020.

doi: 10.21037/atm.2020.02.112

View this article at: http://dx.doi.org/10.21037/atm.2020.02.112

\section{Introduction}

Breast cancer is the most common malignancy in women in Europe, America, as well as in China (1). The incidence of breast cancer among the elderly is higher than that of young people. Cataract is the second leading cause of visual impairment and the first cause of blindness globally. Because ageing is the major risk factor for cataract, the incidence of breast cancer and cataracts will continue to rise with the population aging in China, which would be an important issue in the field of public health (2).

The occurrence of cataract is currently known to be associated with a variety of factors, such as age, genetic susceptibility, diabetes, and drugs such as glucocorticoids, 
chlorpromazine, and mitomycin (3). Estrogen exposure is one of the important causes of breast cancer and about $60-70 \%$ of breast cancers are positive for estrogen receptor (ER). Currently, in patients with ER-positive breast cancer, tamoxifen is usually used in premenopausal patients and the third-generation aromatase inhibitor (AI) is an important option for postmenopausal ones.

Regarding to the relationship between cataract and tamoxifen in breast cancer patients, the current conception is that tamoxifen is a risk factor for cataract, which may be because tamoxifen can alter the metabolism of body lipids (4). Tamoxifen also penetrates the aqueous humor and the vitreous directly, and acts on the crystal (5). The prospective cohort study of NSABP P-01 found that tamoxifen could significantly increase the risk of cataract [hazard ratio (HR) $=1.21$, with $95 \%$ confidence interval (CI): 1.10-1.34] (6). In contrast, there are few studies on the relationship between $\mathrm{AI}$ and subsequent cataract. AI can reduce the blood supply and metabolic abnormalities of the eye because it can lower the estrogen levels (7), which may potentially affect ocular diseases such as cataract and macular degeneration. At present, AI is the mostly used endocrine therapy in postmenopausal patients. We here focused on postmenopausal breast cancer populations to investigate the $\mathrm{AI}$ and cataract risk, by comparing the patients use AI only with those do not use any endocrine therapy. The study population was from the prospectively maintained database of Department of Breast Surgery in Fudan University Shanghai Cancer Center (8). Since its establishment in 2000, the database has been updated and maintained.

\section{Methods}

\section{Breast cancer patients}

We obtained the study cases from the prospectively maintained database from Fudan University Shanghai Cancer Center. The study was approved by the Ethics Committee of the Fudan University Shanghai Cancer Center and each participant signed the informed consent form and agreed to use the anonymity of personal information for clinical research (ID: 0504324-4-201012B). This study included the consecutive postmenopausal breast cancer patients between January 2010 and December 2014.

The inclusion criteria were as followings: (I) postmenopausal women with unilateral, operable, and pathologically confirmed breast carcinoma; (II) having sufficient clinical and pathological information, such as age, tumor size, lymph node status, ER, progesterone receptor (PR), HER2 status, etc.; (III) with records of menstrual status and endocrine therapy drugs as well as endocrine treatment duration; (IV) having information on cataract diseases via follow-up.

The exclusion criteria were: (I) premenopausal or perimenopausal women; (II) women who used tamoxifen [although after 2015, increasing number of premenopausal patients used ovarian function suppression with an AI according to the results from SOFT and TEXT (9), there is insufficient evidence for AI use in premenopausal patients in before 2014]; (III) initially diagnosed stage IV breast cancer or advanced breast cancer disease; (IV) no information on incidence of cataract; (V) confirmed as a familial or agglomerated cataract; (VI) cataract diagnosis before the start of adjuvant $\mathrm{AI}$ treatment.

\section{Immunobistochemistry}

The pathology of ER, PR and HER2 of breast tumors was examined by the Department of Pathology of Fudan University Shanghai Cancer Center. In 2010, ASCO/CAP guidelines established the threshold for ER/PR positive definition to be $1 \%$ (10). HER2 was first tested of the protein expression by immunohistochemistry: 0 and $1+$ were defined as HER2 negative and $3+$ as positive; if $2+$, further fluorescence in situ hybridization ratio (FISH) test is needed.

\section{Endocrine therapy}

If treated with endocrine therapy, patients were administered adjuvant AI. The drug option could be either anastrozole, letrozole, or exemestane. There is currently no evidence of a difference in the efficacy between these three AIs in an adjuvant setting (11). Patients were not allowed to receive tamoxifen, and patients who received tamoxifen with or without an AI was excluded from the study.

\section{Cataract diagnosis}

According to the prevailing standards, the general methods for diagnosing cataract include: (I) symptoms and signs. Through patient's description and doctor's physical examination, the patient has a decrease in lens transparency or opacity, accompanied by visual impairment such as decreased vision and blurred vision; (II) instrument examination, including slit lamp examination, to determine 
Table 1 Characteristics of study patients

\begin{tabular}{lc}
\hline Characteristics & Case $(\mathrm{N}=1,697)$ \\
\hline Age (years) & 140 \\
$<50$ & 1,014 \\
$50-60$ & 543 \\
$\geq 60$ & \\
Tumor size & 853 \\
T0-1 & 844 \\
T2-3 & \\
Grade & 1,106 \\
I-II & 591 \\
III & \\
Lymph node status & \\
Negative & \\
Positive & \\
Estrogen receptor (ER) & \\
Negative & \\
Positive & \\
Progesterone receptor (PR) & \\
Negative & \\
Positive & \\
HER2 & \\
Negative & \\
Positive & \\
\hline
\end{tabular}

the opacity of the lens to determine the extent of the lesion.

During following-up of breast cancer patients in out-patient clinic, for those with complaints such as eye discomfort and blurred vision, they were directly recommended to the Eye Institute of Fudan University Eye and ENT Hospital for further diagnosis. On-site diagnosis of cataracts is subject to the following conditions: visual acuity $<0.7$, lens opacity, and no other eye diseases that cause vision loss. As long as the unilateral eye is diagnosed as a cataract, the patient is diagnosed as the cataract patient. We did not further record the contralateral eye of cataract.

\section{Endpoints and statistics}

The primary study endpoint was the diagnosis of the primary cataract after adjuvant use of AI, and the subgroup analyses were performed for the effect of age and AI treatment duration on cataract. If the breast cancer patients had tumor local and regional recurrence or distant metastasis, the patient will be censored and will not be further followed up for cataract occurrence. If the patient develops cataract during AI or after the finish of $\mathrm{AI}$ treatment, the case is record as a cataract case and the duration of $\mathrm{AI}$ medication was also recorded. If the patient had diagnosed cataract before AI use, the patient should be excluded from this study.

The chi-square test was used for heterogeneity between the different rates, and Fisher method was used for exact chi-square test. The logistic regression was performed for multivariate analysis, and the HR and 95\% CI were calculated. Two-sided $\mathrm{P}<0.05$ was considered statistically significant. Stata v. 13.1 (Stata, College Station, TX) and SPSS 19 (SPSS, Chicago, IL) were used for statistics.

\section{Results}

\section{Basic information of patients}

According to the enrollment criteria, a total of 1,697 postmenopausal women with early-stage breast cancer were included (Table 1). The diagnosis of cataract was updated according to the information from out-patient clinic. If necessary, Eye Institute of Fudan University Eye and ENT Hospital assisted in the diagnosis of cataract. Of the 1,697 patients, 542 were without endocrine therapy and 1,155 were treated with AI alone. Because of the patients were consecutive ones, the age, tumor size, and lymph node status were comparable between the no endocrine group and the AI group. However, the AI group was mainly positive for $\mathrm{ER} / \mathrm{PR}$, and $95 \%$ of the patients without endocrine therapy were ER- and PR-negative. A total of $146(8.6 \%)$ of all patients were diagnosed with cataract during follow-up time.

\section{Association between AI and cataract}

Table 2 shows that the occurrence of cataract is significantly 
Table 2 Association of AI treatment and treatment duration with cataract

\begin{tabular}{|c|c|c|c|c|c|}
\hline Characteristics & \multicolumn{2}{|c|}{ No cataract } & \multicolumn{2}{|c|}{ Cataract } & $P$ \\
\hline \multicolumn{6}{|l|}{ Age (years) } \\
\hline$<50$ & 136 & 97.1 & 4 & 2.9 & $<0.001$ \\
\hline $50-60$ & 944 & 93.1 & 70 & 6.9 & \\
\hline \multicolumn{6}{|l|}{ Use Al or not } \\
\hline No & 502 & 92.6 & 40 & 7.4 & 0.22 \\
\hline Yes* $^{*}$ & 1,049 & 90.8 & 106 & 9.2 & \\
\hline \multicolumn{6}{|l|}{ Al duration } \\
\hline$\geq 5$ years & 29 & 85.3 & 5 & 14.7 & \\
\hline
\end{tabular}

*, we excluded the patients who had received any tamoxifen. Al, aromatase inhibitor.

related to age. Patients aged $<50,50-60$, and $\geq 60$ years have a gradual increase in the risk of cataract, with the incidence rates of $2.9 \%, 6.9 \%$, and $13.3 \%$, respectively $(\mathrm{P}<0.001)$. However, the use of adjuvant AI did not appear to be associated with cataract $(7.4 \%$ vs. $9.2 \%, \mathrm{P}=0.22)$. The other clinicopathological factors were not associated with cataract risk.

\section{AI treatment duration and cataract}

Although use AI or not had no significant association with the occurrence of cataract, the risk of cataract could also be affected by the duration of medication. We found that the incidence of cataract was similar in patients who used AI within 3 and $3-5$ years (9.5\% and $9.0 \%$, respectively). For those with longer application of AI more than 5 years, $14.7 \%$ were diagnosed with cataracts, however the difference was only numerical but not statistical $(\mathrm{P}=0.52)$.

\section{Multivariate analysis of cataract}

After adjusting for potential confounders such as patient age, tumor size, and lymph node status (Table 3), we found that $\mathrm{AI}$ was not an independent risk factor for cataract (HR $=1.20$; $95 \%$ CI: $0.8-1.7, \mathrm{P}=0.30$ ). Age is a very profound independent factor. Elderly patients older than 60 years had 5 times cataract risk compared to younger patients regardless of endocrine therapy or not.

\section{Discussion}

AI can prevent the conversion of androgen to estrogen by inhibiting aromatase, resulted in reduced estrogen levels. $\mathrm{AI}$ is an important choice for adjuvant endocrine therapy in early postmenopausal ER/PR positive breast cancer. In this study, we found that AI was not related to cataract risk in the study population. For patients with prolonged AI treatment, the incidence of cataract numerically but not statistically increased. In contrast, age was an important risk factor of cataract. It indicates that in the long-term followup of breast cancer patients, the risk of ocular diseases such as cataract should be paid close attention to.

Thus far, there are a number of literatures on tamoxifen and cataracts (12). In patients with breast cancer taking tamoxifen, the incidence of cataract was $18.2 \%$ and $21.4 \%$ for those having $4-5$ years and those having more than 6 years, respectively. However, the incidence of cataract was only about $5 \%$ in the untreated group. The prospective clinical trial NSABP P-01 also suggested that prophylactic use of tamoxifen significantly increased the risk of cataract (risk ratio of 1.21 , with $95 \%$ CI: $1.10-1.34$ ) (6). The mechanism of AI is not the same as tamoxifen. Therefore, the relationship between $\mathrm{AI}$ and cataract is worth of further investigation. Some previously reported studies had described 
Table 3 Multivariate analysis of risk factors for cataract

\begin{tabular}{|c|c|c|c|}
\hline Clinical and pathological parameters & $\mathrm{HR}$ & $95 \% \mathrm{Cl}$ & $P$ value \\
\hline$\leq 50$ & Reference & & \\
\hline $50-60$ & 2.57 & $0.92-7.1$ & 0.07 \\
\hline$\geq 60$ & 5.20 & $1.9-14.4$ & 0.002 \\
\hline No & Reference & & \\
\hline Yes & 1.20 & $0.8-1.7$ & 0.30 \\
\hline \multicolumn{4}{|l|}{ Tumor size } \\
\hline T0-1 & Reference & & \\
\hline$I-I I$ & Reference & & \\
\hline III & 0.74 & $0.5-1.1$ & 0.19 \\
\hline \multicolumn{4}{|l|}{ Lymph node status } \\
\hline \multicolumn{4}{|l|}{ Yes } \\
\hline No & 0.73 & $0.5-1.1$ & 0.12 \\
\hline
\end{tabular}

toxic side effects of exemestane such as cataracts (13). However, these studies have some limits: first, the sample size is too small to have a powerful statistical analysis; second, the length of AI treatment is short and insufficient, with many confounding factors; third, it did not exclude naturally age-related cataract. Therefore, a larger sample size study with prospectively maintained data is needed to determine the cataract risk of AI. Our study is the first large sample (more than 1,500 cases), confirming that there is no significant relationship between AI and cataract in general population.

The advantage of this study is the use of prospectively maintained database. We can get the accurate followup information and the results are more credible. We also exclude the cases which had received tamoxifen to reduce the confounding risk. Our study also had a few limits. First, we cannot analyze impact of chemotherapy on cataract risk, since some study indicated chemotherapy or radiotherapy might be associated with ocular diseases. Second, we here did not distinguish different type of AI, steroidal or nonsteroidal. Although the efficacy of the three AIs (anastrozole, letrozole, exemestane) is currently consistent, the toxicities on ocular diseases is yet uncertain.
Third, the enrolled population was highly screened and the results needed further validation. The absolute value of the incidence of cataract in our study is relatively lower than another epidemiological report in China (2). Interestingly, the prospective clinical cohort studies such as NSABP P-01 have an even lower incidence of cataract, about 2-3\% (6). The underlying cause is that the patients enrolled in our study are postmenopausal and elder women. In addition, during our clinical follow-up, patients with "self-reported visual discomfort" were referred to the otolaryngology hospital for further investigation, which greatly increased the detection rate of cataract.

In summary, we have demonstrated for the first time that there is no significant association between cataract occurrence and adjuvant $\mathrm{AI}$ in postmenopausal early breast cancer patients. Of note, age is an important risk factor for cataracts. Regardless of the presence or absence of AI medication, older patients should pay more attention to their eye health, especially the risk and diagnosis of cataracts.

\section{Acknowledgments}

Funding: Supported by grants from the National Natural 
Science Foundation of China (81672600, and 81722032), 2018 Shanghai Youth Excellent Academic Leader, and Fudan Zhuoshi Project. Study on comprehensive prevention and control of common eye diseases in Xuhui District (XHLHGG201807), and Shanghai Key Laboratory of Visual Impairment and Restoration.

\section{Footnote}

Conflicts of Interest: The authors have no conflicts of interest to declare.

Ethical Statement: The authors are accountable for all aspects of the work in ensuring that questions related to the accuracy or integrity of any part of the work are appropriately investigated and resolved. The study was approved by the Ethics Committee of the Fudan University Shanghai Cancer Center and each participant signed the informed consent form and agreed to use the anonymity of personal information for clinical research (ID: 0504324-4201012B).

Open Access Statement: This is an Open Access article distributed in accordance with the Creative Commons Attribution-NonCommercial-NoDerivs 4.0 International License (CC BY-NC-ND 4.0), which permits the noncommercial replication and distribution of the article with the strict proviso that no changes or edits are made and the original work is properly cited (including links to both the formal publication through the relevant DOI and the license). See: https://creativecommons.org/licenses/by-nc-nd/4.0/.

\section{References}

1. Fan L, Strasser-Weippl K, Li JJ, et al. Breast cancer in China. Lancet Oncol 2014;15:e279-89.

2. Song P, Wang H, Theodoratou E, et al. The national and subnational prevalence of cataract and cataract blindness in China: a systematic review and meta-analysis. J Glob Health 2018;8:010804.

Cite this article as: Chen $\mathrm{H}$, Shao ZM, Yu KD, Xu GZ. Association of adjuvant aromatase inhibitor with cataract risk in postmenopausal women with breast cancer. Ann Transl Med 2020;8(6):342. doi: 10.21037/atm.2020.02.112
3. Eisner A, Luoh SW. Breast cancer medications and vision: effects of treatments for early-stage disease. Curr Eye Res 2011;36:867-85.

4. Zhang JJ, Jacob TJ, Valverde MA, et al. Tamoxifen blocks chloride channels. A possible mechanism for cataract formation. J Clin Invest 1994;94:1690-7.

5. Kirker MR, Gallagher KM, Witt-Enderby PA, et al. High affinity nuclear and nongenomic estradiol binding sites in the human and mouse lens. Exp Eye Res 2013;112:1-9.

6. Fisher B, Costantino JP, Wickerham DL, et al. Tamoxifen for the prevention of breast cancer: current status of the National Surgical Adjuvant Breast and Bowel Project P-1 study. J Natl Cancer Inst 2005;97:1652-62.

7. Howell A, Cuzick J. Vascular effects of aromatase inhibitors: data from clinical trials. J Steroid Biochem Mol Biol 2005;95:143-9.

8. Yu KD, Di GH, Wu J, et al. Development and trends of surgical modalities for breast cancer in China: a review of 16-year data. Ann Surg Oncol 2007;14:2502-9.

9. Francis PA, Pagani O, Fleming GF, et al. Tailoring Adjuvant Endocrine Therapy for Premenopausal Breast Cancer. N Engl J Med 2018;379:122-37.

10. Hammond ME, Hayes DF, Dowsett M, et al. American Society of Clinical Oncology/College Of American Pathologists guideline recommendations for immunohistochemical testing of estrogen and progesterone receptors in breast cancer. J Clin Oncol 2010;28:2784-95.

11. Early Breast Cancer Trialists' Collaborative Group (EBCTCG). Aromatase inhibitors versus tamoxifen in early breast cancer: patient-level meta-analysis of the randomised trials. Lancet 2015;386:1341-52.

12. Paganini-Hill A, Clark LJ. Eye problems in breast cancer patients treated with tamoxifen. Breast Cancer Res Treat 2000;60:167-72.

13. Iwata H, Masuda N, Ohno S, et al. A randomized, doubleblind, controlled study of exemestane versus anastrozole for the first-line treatment of postmenopausal Japanese women with hormone-receptor-positive advanced breast cancer. Breast Cancer Res Treat 2013;139:441-51. 